\title{
A Diffusion Model for Growth Stocks
}

\author{
S. C. Kou \\ Department of Statistics, Science Center 603, Harvard University, Cambridge, \\ Massachusetts 02138, kou@stat.harvard.edu \\ S. G. Kou \\ Department of IEOR, 312 Mudd Building, Columbia University, New York, \\ New York 10027,sk75@columbia.edu
}

\begin{abstract}
Since growth stocks tend to have low or even negative earnings and high volatility, it is a great challenge to derive a meaningful mathematical model within the traditional valuation framework. This paper attempts to derive a suitable diffusion model for growth stocks by using the idea of size distribution. Numerical illustration of the model based on the data covering the time period of the recent boom and burst of the "Internet bubble" is also presented.

Key words: Feller process; CIR model; power law; steady-state distribution; weak convergence; decay rate; regression

MSC2000 subject classification: Primary: 60H30, 91B70

OR/MS subject classification: Primary: Diffusion, asset pricing

History: Received July 23, 2002; revised June 26, 2003.
\end{abstract}

1. Introduction and background. The recent boom and burst of the "Internet bubble" present some interesting questions and challenges for financial modeling. First, are growth stocks still relevant and important enough to study? Second, since growth stocks tend to have low or even negative earnings and high volatility, traditional valuation framework, such as the net present value method, cannot be used; a question is then how to derive a meaningful mathematical model, if possible, for growth stocks. Third, any reasonable model must incorporate the "boom and burst" phenomenon.

For the first question, it is important to note that although the components of growth stocks may vary over time (perhaps consisting of railroad and utility stocks in the early 1900s, and biotechnology and Internet stocks in 2003), studying their general properties is essential to understanding financial markets and economic growth in the past, at present, and perhaps in the future too.

The goal of the current paper is to provide a model to answer the other two questions. To do so, we present a continuous-time diffusion model for growth stocks. The model extends the discrete model, which is based on a birth-death process, in Kou and Kou (2003).

The discrete model gives a relative pricing formula for growth stocks, and only uses the unique feature of high volatility of growth stocks. Neither earnings (which are not available for most growth stocks), nor forecasted earnings (which not only are highly unreliable, as evident in the recent events related to the "Internet bubble," but also lack clear mathematical relationships with stock values) are used in the model. The discrete model also shed light on an empirically observed puzzle that there is an "almost" linear relationship between the logarithm of the market capitalization of growth stocks and the logarithm of their associated ranks (which was first reported in the Wall Street Journal, Dec. 27, 1999, p. C1, Column 1 and p. C2, Column 3, only for Internet stocks), and that at the same time this phenomenon, more interestingly, did not seem to hold for nongrowth stocks. Translating into a probabilistic language, this empirical puzzle means that the size distribution of the growth stocks almost follows a power law, and it is not so for ordinary stocks.

To introduce our continuous-time diffusion model for growth stocks, we first briefly review the discrete model, which relies on a linear birth-death process with immigration and emigration. Suppose at time $t$ a growth stock has total market capitalization $T(t)$. The discrete model postulates that $T(t)=\Theta(t) X(t)$, where $\Theta(t)$ represents the overall economic 
and sector trend, and $X(t)$ represents individual stock variation within the sector, so $\Theta(t)$ is the same for all firms within the same industry sector, and $X(t)$ varies from firm to firm. The individual variation term $X(t)$ in the discrete model is posed as a linear birth-death process: Given $X(t)$ being in state $i, i=0,1,2, \ldots$ (the unit of $X(t)$ could be, for example, millions or billions of dollars), the instantaneous movements are: (a) $i \rightarrow i+1$, with rate $i \lambda+g$, $i \geq 0$, and (b) $i \rightarrow i-1$, with rate $i \mu+h, i \geq 1$. In other words, the individual variation term $X(t)$ follows a linear birth-death process with the birth rates $\lambda_{i}=i \lambda+g(i \geq 0)$ and the death rates $\mu_{i}=i \mu+h(i \geq 1), \mu_{0}=0$. In the discrete model, the two parameters $\lambda$ and $\mu$ represent the instantaneous appreciation and depreciation rates of $X(t)$ due to market fluctuations; the model assumes that they influence the market capitalization proportionally to the current value. The requirement $\lambda<\mu$ is postulated in the discrete model to ensure that the birth-death process has a steady-state distribution. The parameter $g$ models the rate of increase in $X(t)$ due to nonmarket factors, such as the effect of additional shares being issued through public offerings, or the effect of warranties on the stock being exercised (resulting in new shares being issued). The parameter $h$ models the rate of decrease in $X(t)$ due to nonmarket factors, such as the dividend payment; for most growth stocks $h \approx 0$, as no dividends are paid.

In the steady state the discrete model leads to an almost linear curve for stocks with high volatility (such as biotechnology and Internet stocks) when the log-market-capitalization is plotted against the log-rank; at the same time the model implies that for nongrowth stocks such a phenomenon should not be expected, primarily because of the very slow convergence of the birth-death process to its steady state distribution due to a low volatility. Furthermore, the discrete model also suggests a way to relatively price growth stocks, not just Internet stocks (as the Wall Street Journal article focused).

In this paper, to construct a continuous-time diffusion model for growth stocks, we first consider the weak (convergence) limit of the linear birth-death process in the discrete model; then, guided by the limit, we investigate, rigorously, a general class of diffusion processes and identify suitable ones that can fit the observed size distribution for Internet and biotechnology stocks. The model, thus, shows that a diffusion model for growth stocks can be built mainly by utilizing the high volatility of their share prices. Since the model also contains a mean reversion component, it may be useful in understanding the recent boom and burst of the "Internet bubble."

The size distribution of growth stocks plays an important role in our model. We shall point out that studying power law and size distribution has a long history, dating back at least to Pareto (1896), Yule (1924), Zipf (1949), Simon (1955), and Ijiri and Simon (1977). It is interesting to see that such a classical probabilistic subject can still provide useful insight for today's high-technology markets.

The paper is organized as follows. Section 2.1 provides the weak convergence result, motivating us to consider a class of diffusion models in $\$ 2.2$. The properties of the model are studied in $\S 3$. Based on these properties, size distributions are analyzed in $\S 4$. Section 5 specializes to the size distribution of biotechnology and Internet stocks and discusses relative pricing of growth stocks. Some numerical illustrations are given in §6. The last section offers some discussion. The proofs are deferred to the appendix.

2. The model. This section presents a continuous-time diffusion model for growth stocks. Instead of working on the price of a growth stock, it makes more sense to study market capitalization, defined as the product of the total outstanding shares and the market price of the stock, because growth stocks tend to have frequent stock splits, which immediately drops the price significantly but has little effect on market capitalization.

2.1. From the discrete model to a continuous model. An intuitive way to derive continuous diffusion models for growth stocks is to consider the limit of the discrete model. Note that in the discrete model the jump size of the linear birth-death process $X(t)$ is 1 and 
the infinitesimal increment $d X_{t}$ satisfies

$$
d X(t)= \begin{cases}1 & \text { with probability }(\lambda X(t)+g) d t+o(d t) \\ -1 & \text { with probability }(\mu X(t)+h) d t+o(d t) \\ 0 & \text { otherwise }\end{cases}
$$

Now if we let the jump size be $\Delta s$, then the birth-death process becomes

$$
d X(t)= \begin{cases}\Delta s & \text { with probabibility }(\lambda X(t)+g) d t+o(d t), \\ -\Delta s & \text { with probabibility }(\mu X(t)+h) d t+o(d t), \\ 0 & \text { otherwise. }\end{cases}
$$

The conditional mean and variance of $d X(t)$ are, respectively, $[(\lambda-\mu) X(t)+(g-h)]$. $\Delta s d t+o(d t)$ and $\left[(\lambda+\mu) X_{t}+(g+h)\right] \Delta s^{2} d t+o(d t)$. Thus, if we let $\Delta s \rightarrow 0$ in such a way that

$$
\begin{array}{cl}
(\lambda-\mu) \Delta s \rightarrow-b \sigma^{2}<0, & (g-h) \Delta s \rightarrow a \sigma^{2}>0, \\
(\lambda+\mu) \Delta s^{2} \rightarrow \sigma^{2}, & (g+h) \Delta s^{2} \rightarrow 0,
\end{array}
$$

then the limiting process will satisfy

$$
\begin{gathered}
E\left(d X(t) \mid \mathscr{F}_{t}\right)=\left(-b \sigma^{2} X(t)+a \sigma^{2}\right) d t+o(d t), \\
\operatorname{Var}\left(d X(t) \mid \mathscr{F}_{t}\right)=\sigma^{2} X(t) d t+o(d t) .
\end{gathered}
$$

(Note that in many cases since the parameter $h$, which represents the dividend payment, is zero, the normalization condition $(g-h) \Delta s \rightarrow a \sigma^{2}$ automatically implies another condition $(g+h) \Delta s^{2} \rightarrow 0$ above.) With this parameterization, as $\Delta s \downarrow 0$, intuitively the birth-death process $X(t)$ seems to converge to a limiting diffusion process that satisfies the stochastic differential equation

$$
d X(t)=\left(-b \sigma^{2} X(t)+a \sigma^{2}\right) d t+\sigma \sqrt{X(t)} d W(t), \quad X(0)=x,
$$

where $W(t)$ is a standard Brownian motion. The following theorem makes this intuition rigorous.

THEOREM 2.1. Introduce a sequence of birth-death processes $X_{n}(t), n \geq 1$, with $X_{n}(0)=$ $\lfloor n x\rfloor / n \geq 0$, where $x \geq 0$, and state space of $\left\{X_{n}(t) ; t \geq 0\right\}$ being $E_{n}=\{0,1 / n, 2 / n, \ldots\}$; i.e., the ith state of $\left\{X_{n}(t) ; t \geq 0\right\}$ is $i / n$. Let the birth rate and death rate of $X_{n}(t)$ at the ith state be $\lambda_{n}(i / n)+g_{n}, i \geq 0$, and $\mu_{n}(i / n)+h_{n}, i \geq 1$, respectively. Choose $\lambda_{n}, \mu_{n}, g_{n}$, and $h_{n}$ in such a way that $\lambda_{n}<\mu_{n}$ and as $n \rightarrow \infty$,

$$
\begin{aligned}
\frac{\lambda_{n}-\mu_{n}}{n} \rightarrow-b \sigma^{2}<0, & \frac{g_{n}-h_{n}}{n} \rightarrow a \sigma^{2}>0, \\
\frac{\lambda_{n}+\mu_{n}}{n^{2}} \rightarrow \sigma^{2}, & \frac{g_{n}+h_{n}}{n^{2}} \rightarrow 0 .
\end{aligned}
$$

Then $X_{n}(t) \Rightarrow X(t)$ on $D[0, \infty)$ (the space of all random functions on $[0, \infty]$ that are right continuous with left limit existing) with Stone's topology.

Proof. See Appendix A.

\subsection{Formulation of the model.}

The Model. Theorem 2.1 motivates us to consider a general model: the total market capitalization

$$
T(t)=\Theta(t) X(t)
$$


where $\Theta(t)$ represents the overall economic and sector trend, and $X(t)$ represents individual stock variation, which follows the diffusion process

$$
d X(t)=\left(-b \sigma^{2} X(t)+a \sigma^{2}\right) d t+\sigma X^{\gamma}(t) d W(t), \quad X(0)=x>0, \gamma>0 .
$$

The parameters in the model have their own economic interpretations: $\sigma$ corresponds to the stock volatility; the parameter $a$, as suggested by the limiting relationship (2.1), models the money inflow to the market capitalization due to nonmarket factors such as exercising of employee warranties and public offering of new/additional shares, etc.; $a / b$ is the mean reverting level of $X(t)$ (which implies that the market capitalization $T(t)$ is mean reversion toward the overall economic trend $\Theta(t))$. Note that when $\gamma=\frac{1}{2}$, we have the limiting diffusion process in Theorem 2.1.

Of course, without the previous model based on the birth-death process, it would be very difficult to imagine a model like (2.3). Therefore, the discrete birth-death process provides a nice intuition for the continuous diffusion models. (Furthermore, the discrete model is perhaps more suitable for modeling intra-day tick-by-tick data.) Although, as we shall see, the steady state distributions of the discrete and continuous models are similar (thus, the size distributions are similar), the diffusion model has its own merits, which make it advantageous over the discrete model: (1) Generally speaking, diffusion models can lead to many closed form solutions, whence they have better analytical tractability than birthdeath processes. (2) It is possible to do riskless hedging for diffusion models, while this is impossible for many discrete models.

An interesting feature of (2.3) is that it has mean reversion in $X(t)$, which is quite unusual for models of stocks. However, since the market cap $T(t)=\Theta(t) X(t)$, we note that the mean reversion is actually reversion toward the overall economic trend $\Theta(t)$. Thus, although in the long run the market cap would move upward (as $\Theta(t)$ may do), in a relatively short time period one could observe the mean reverting phenomenon. Thus, the mean reversion feature of the model may also provide some interesting insight about the recent boom and burst of the "Internet bubble"- "What goes up must come down eventually."

To study the cross-sectional size distribution, which is the focus of the current paper, it is not necessary to model $\Theta(t)$ explicitly, except by assuming that $\Theta(t)$ is bounded away from 0 . However, modeling $\Theta(t)$ explicitly may lead to some dynamic implications, rather than just cross-sectional implications. Indeed, it can be shown (Kou and Kou 2002) that the dynamics of the model are also linked to the stochastic endogenous growth theory in macroeconomics.

3. Properties of the diffusion process $X(t)$. Although the diffusion process (2.3) has been used in economics to study interest rates, a rigorous treatment of Equation (2.3) for the whole range of $0<\gamma<\infty$ is missing in the literature, except for the two special cases of $\gamma=1$ (Wong Process, Wong 1964) and $\gamma=\frac{1}{2}$ (Feller process, Feller 1951). In particular, as we shall see in Theorem 3.1, when $0<\gamma \leq \frac{1}{2}$, since 0 may be a regular boundary, the stochastic differential equation (2.3) may not have a unique solution (as the global Lipschitz and growth conditions for the uniqueness may fail to hold). Indeed, as we shall see in Theorem 3.1, when $0<\gamma \leq \frac{1}{2}$, a proper boundary condition, such as a reflecting boundary at 0 (see, e.g., Mandl 1968, p. 67, and Chapter 8 in Chung and Williams 1990), must be imposed to ensure that the solution is unique and that the steady-state distribution exists.

Our rigorous treatment of the diffusion process (2.3) uses the results in Mandl (1968). The first step is to characterize the boundary behavior of the diffusion (2.3) at 0 and $\infty$. (Note that there are four possible types of boundary behavior: regular, natural, entrance, and exit; see Karlin and Taylor 1981, §§15.7 and 15.8.)

Following Karlin and Taylor (1981, p. 221 and §15.6), for a general time-homogeneous diffusion process with drift function $\mu(x)$ and volatility function $\sigma^{2}(x)$, to study the bound- 
Kou and Kou: A Diffusion Model for Growth Stocks

ary behavior, the scale density $s(x)$ and scale measure $S(x)$ are introduced as

$$
\begin{gathered}
s(x):=\exp \left(-\int^{x} \frac{2 \mu(y)}{\sigma^{2}(y)} d y\right), \quad S(x):=\int^{x} s(\eta) d \eta, \\
S[\alpha, \beta]:=S(\beta)-S(\alpha), \quad S(\alpha, \beta]:=S(\beta)-S(\alpha+), \quad S[\alpha, \beta):=S(\beta-)-S(\alpha),
\end{gathered}
$$

where $\int^{x}$ denotes indefinite integral. We also introduce the speed density and speed measure

$$
\begin{aligned}
m(x) & :=\frac{1}{\sigma^{2}(x) s(x)}, \\
M[\alpha, \beta]:=\int_{\alpha}^{\beta} m(x) d x, \quad M[\alpha, \beta) & :=\lim _{x \rightarrow \beta-} M[\alpha, x], \quad M(\alpha, \beta]:=\lim _{x \rightarrow \alpha+} M[x, \beta],
\end{aligned}
$$

and furthermore,

$$
N(0,1]:=\int_{0}^{1}\left[\int_{\eta}^{1} s(\xi) d \xi\right] m(\eta) d \eta, \quad N[1, \infty):=\int_{x}^{\infty}\left[\int_{x}^{\eta} s(\xi) d \xi\right] m(\eta) d \eta .
$$

(See Karlin and Taylor 1981, pp. 231, 237.)

In our diffusion equation (2.3), the drift and volatility are, respectively, $\mu(x)=-b \sigma^{2} x+$ $a \sigma^{2}, \sigma^{2}(x)=\sigma^{2} x^{2 \gamma}$. It follows that

$$
s(x)=\exp \left\{-2 \int^{x} \frac{-b y+a}{y^{2 \gamma}} d y\right\} .
$$

Depending on the value of $\gamma, s(x)$ has three possible forms:

(a) $\gamma \neq 1, \frac{1}{2}$, in which case,

$$
s(x)=\exp \left\{-2\left(\frac{b}{2 \gamma-2} x^{2-2 \gamma}-\frac{a}{2 \gamma-1} x^{1-2 \gamma}\right)\right\} .
$$

\begin{tabular}{|c|c|c|c|c|c|c|}
\hline & $\gamma>1$ & $\gamma=1$ & $\frac{1}{2}<\gamma<1$ & $\begin{array}{c}\gamma=\frac{1}{2} \text { and } \\
a>\frac{1}{2}\end{array}$ & $\begin{array}{l}\gamma=\frac{1}{2} \text { and } \\
0<a \leq \frac{1}{2}\end{array}$ & $0<\gamma<\frac{1}{2}$ \\
\hline $\lim \sigma^{2}(x) s(x)$ & $\infty$ & $\infty$ & $\infty$ & $\infty$ & 0 & 0 \\
\hline $\lim _{x \rightarrow 0+} S(x)$ & 1 & 1 & 1 & 1 & & \\
\hline $\lim _{x \rightarrow 0+} \overline{\sigma^{2}(x) s(x)}$ & $\overline{2 a \sigma^{2}}$ & $\overline{2 a \sigma^{2}}$ & $\overline{2 a \sigma^{2}}$ & $\overline{(2 a-1) \sigma^{2}}$ & $-\infty$ & 0 \\
\hline$S(0, x]$ & $\infty$ & $\infty$ & $\infty$ & $\infty$ & $<\infty$ & $<\infty$ \\
\hline$M(0, x]$ & $<\infty$ & $<\infty$ & $<\infty$ & $<\infty$ & $<\infty$ & $<\infty$ \\
\hline$N(0,1]$ & $<\infty$ & $<\infty$ & $<\infty$ & $<\infty$ & $<\infty$ & $<\infty$ \\
\hline $\lim _{x \rightarrow \infty} \frac{\sigma^{2}(x) s(x)}{x}$ & $\infty$ & $\infty$ & $\infty$ & $\infty$ & $\infty$ & $\infty$ \\
\hline$S(x)$ & 0 & 1 & 1 & 1 & 1 & 1 \\
\hline $\lim _{x \rightarrow \infty} \frac{}{\sigma^{2}(x) s(x) / x}$ & 0 & $\overline{(2 b+1) \sigma^{2}}$ & $\overline{2 b \sigma^{2}}$ & $\overline{2 b \sigma^{2}}$ & $\overline{2 b \sigma^{2}}$ & $\overline{2 b \sigma^{2}}$ \\
\hline$S[x, \infty)$ & $\infty$ & $\infty$ & $\infty$ & $\infty$ & $\infty$ & $\infty$ \\
\hline$M[x, \infty)$ & $<\infty$ & $<\infty$ & $<\infty$ & $<\infty$ & $<\infty$ & $<\infty$ \\
\hline$N[1, \infty)$ & $<\infty$ & $\infty$ & $\infty$ & $\infty$ & $\infty$ & $\infty$ \\
\hline
\end{tabular}

(b) $\gamma=\frac{1}{2}$, which corresponds to the Feller process, and $s(x)=e^{2 b x} x^{-2 a}$.

(c) $\gamma=1$, under which $s(x)=x^{2 b} e^{2 a / x}$.

To classify the boundary behavior as well as to study the steady-state distribution, we need the following lemma.

Lemma 3.1. For the diffusion process (2.3), the following table gives various limiting results. 
Proof. See Appendix A.

Based on this lemma, we have the following result.

THEOREM 3.1. (i) The boundaries 0 and $\infty$ of the diffusion (2.3) are classified as follows:

\begin{tabular}{lll}
\hline & \multicolumn{1}{c}{0} & \multicolumn{1}{c}{$\infty$} \\
\hline (A) $\gamma>1$ & entrance & entrance \\
(B) $\gamma=1$ & entrance & natural \\
(C) $\frac{1}{2}<\gamma<1$ & entrance & natural \\
(D) $\gamma=\frac{1}{2}$ and $a>\frac{1}{2}$ & entrance & natural \\
(E) $\gamma=\frac{1}{2}$ and $0<a \leq \frac{1}{2}$ & regular & natural \\
(F) $0<\gamma<\frac{1}{2}$ & regular & natural \\
\hline
\end{tabular}

(ii) For the cases of (A) $\gamma>1$, (B) $\gamma=1$, (C) $\frac{1}{2}<\gamma<1$, and (D) $\gamma=\frac{1}{2}$ and $a>\frac{1}{2}$, the solution of (2.3) is unique. Furthermore, as $t \rightarrow \infty$, the diffusion (2.3) has a unique steady-state distribution independent of the initial position $X(0) \in(0, \infty)$. The steady-state density $f(x)$ is given by

$$
f(x)=\frac{C_{2}}{\sigma^{2}(x) s(x)}
$$

where the normalizing constant $C_{2}$ makes $\int_{0}^{\infty} f(x)=1$.

(iii) For the cases of (E) $\gamma=\frac{1}{2}$ and $0<a \leq \frac{1}{2}$, and (F) $0<\gamma<\frac{1}{2}$, because 0 is a regular boundary, the solution of (2.3) is not unique and depends on how we impose additional boundary constraints. If we assume that 0 is a reflecting boundary (similar to the discrete birth-death model), then the solution of (2.3) is unique, and as $t \rightarrow \infty$ the process (2.3) also has a unique steady-state distribution independent of the initial position $X(0) \in(0, \infty)$. The steady-state density $f(x)$ is again given by

$$
f(x)=\frac{C_{2}}{\sigma^{2}(x) s(x)},
$$

where $C_{2}$ is a normalizing constant so that $\int_{0}^{\infty} f(x)=1$.

Proof. See Appendix A.

The following theorem calculates the steady-state distribution of $X(t)$ and gives the asymptotic tail behavior of the steady-state distribution that will be used later.

THEOREM 3.2. Let $f(x)$ denote the density of the steady-state distribution.

(1) When $\gamma>1$, we have

$$
f(x)=C_{2} x^{-2 \gamma} \exp \left\{2\left(\frac{b}{2 \gamma-2} x^{2-2 \gamma}-\frac{a}{2 \gamma-1} x^{1-2 \gamma}\right)\right\} .
$$

The tail probability $F(z):=\mathrm{P}(X(\infty)>z)$ asymptotically satisfies $F(z) \cong C z^{1-2 \gamma}$, as $z \rightarrow \infty$, where $C_{2}$ and $C$ are two normalizing constants, and the notation $a \cong b$ means $\lim a / b=1$.

(2) When $\gamma=1$, we have $f(x)=C_{2} x^{-2(1+b)} e^{-2 a / x}$, and the tail probability $F(z) \cong$ $C z^{-1-2 b}$, for some constants $C_{2}$ and $C$.

(3) When $\frac{1}{2}<\gamma<1$,

$$
f(x)=C_{2} x^{-2 \gamma} \exp \left(-\frac{b}{1-\gamma} x^{2-2 \gamma}\right) \exp \left(-\frac{2 a}{2 \gamma-1} x^{1-2 \gamma}\right),
$$

with the tail probability $F(z) \cong C z^{-1} \exp \left((b /(\gamma-1)) z^{2-2 \gamma}\right)$, for some constants $C_{2}$ and $C$.

(4) When $\gamma=\frac{1}{2}$ and with 0 being a reflecting boundary if $0<a \leq \frac{1}{2}$, we have $f(x)=$ $C_{2} e^{-2 b x} x^{2 a-1}$, and the tail probability $F(z) \cong C e^{-2 b z} z^{2 a-1}, C_{2}=C=(2 b)^{2 a} / \Gamma(2 a)$. 
(5) When $0<\gamma<\frac{1}{2}$ and with 0 being a reflecting boundary, the density $f(x)$ and tail probability $F(z)$ satisfy

$$
\begin{aligned}
& f(x)=C_{2} x^{-2 \gamma} \exp \left(-\frac{b}{1-\gamma} x^{2-2 \gamma}\right) \exp \left(-\frac{2 a}{2 \gamma-1} x^{1-2 \gamma}\right), \\
& F(z) \cong C z^{-1} \exp \left(-\frac{b}{1-\gamma} z^{2-2 \gamma}\right) \exp \left(-\frac{2 a}{2 \gamma-1} z^{1-2 \gamma}\right),
\end{aligned}
$$

for some constants $C_{2}$ and $C$.

Proof. See Appendix A.

4. General properties of size distribution. Consider $N$ (an unknown quantity, may be very large) growth firms within a particular sector governed by the diffusion model, among which the $K$ largest ones (in terms of their market capitalization) are included in a group to be studied. Denote the market capitalization of the $K$ observed stocks by $T_{i}(t), 1 \leq i \leq K$. Since all these $K$ firms are from the same sector, we have

$$
T_{i}(t)=\Theta(t) X_{i}(t),
$$

where $\Theta(t)$, the overall economic and sector trend, is the same for all $K$ stocks, but the individual variation terms $X_{i}(t)$ are different.

Now suppose we rank the market capitalizations such that $T_{(1)}(t) \geq T_{(2)}(t) \geq \cdots \geq T_{(K)}(t)$, where $T_{(1)}(t)$ denotes the largest firm, $T_{(2)}(t)$ the second largest firm, etc. Then we have

$$
\log T_{(i)}(t)=\log \Theta(t)+\log \left(X_{(i)}(t)\right),
$$

where $X_{(i)}(t)$ are the ranked values of $X_{i}(t), 1 \leq i \leq K$. Since the first term $\Theta$ in (4.1) is common for all firms, the plot of $\log T_{(i)}(t)$ versus $\log i$ and the plot of $\log X_{(i)}(t)$ versus $\log i$ will display similar patterns. Therefore, we first focus on the relationship between $\log X_{(i)}(t)$ and $\log i$.

As $X_{(1)}(t), X_{(2)}(t), \ldots, X_{(K)}(t)$ are the ordered realizations of $X(t)$, the empirical tail distribution $\widetilde{F}(x)$ (the empirical version of $F$ ) evaluated at $X_{(i)}(t)$ is simply $\tilde{F}\left(X_{(i)}(t)\right)=$ $i / N, i=1, \ldots, K$. Now assume

(A1): The diffusion process has reached the steady state.

(A2): For each stock in the group, the market capitalization is large; even $X_{(K)}$ is large.

Then we can apply the result of Theorem 3.2 to study the size distribution of growth stocks. It is worth noting that Assumption (A2) implies that the model is only valid for large-cap growth stocks. To study the size distribution of growth stocks we need to discuss five cases.

(1) $\gamma>1$. According to Theorem 3.2, in the steady state, for large $z, \log F(z) \cong C+$ $(1-2 \gamma) \log z$, for some constant $C$. Therefore, empirically, we shall expect that

$$
\log (i / N)=\log \tilde{F}\left(X_{(i)}\right) \approx \log F\left(X_{(i)}\right) \cong C+(1-2 \gamma) \log X_{(i)} .
$$

The notation " $\approx$ " here indicates that we want to use the empirical distribution to approximate the true distribution. This is valid if the total sample size $N$ is large enough and if $X_{i}(t)$ are independent (note that $T_{i}(t)$ are still dependent due to $\Theta(t)$ ); of course, the approximation may still be valid if the dependence among $X_{i}(t)$ is not significant so that ergodic-type limiting theorems hold. Rearranging the terms in (4.2) yields

$$
\log X_{(i)} \approx C_{N}-\frac{1}{2 \gamma-1} \log i,
$$

for some constant $C_{N}$ that depends on $N$. Hence, the slope of the size distribution (i.e., the slope of regressing $\log X_{(i)}$ on $\left.\log i\right)$ is $-1 /(2 \gamma-1)$. 
(2) $\gamma=1$. In this case $\log F(z) \cong C-(1+2 b) \log z$. The empirical version is, therefore, $\log (i / N) \approx C-(1+2 b) \log X_{(i)}$. Or equivalently,

$$
\log X_{(i)} \approx C_{N}-\frac{1}{1+2 b} \log i
$$

So the slope of the size distribution is $-1 /(1+2 b)$.

(3) $\frac{1}{2}<\gamma<1$. According to Theorem 3.2, $\log F(z) \cong C-\log (z)-(b /(1-\gamma)) z^{2-2 \gamma}$. The empirical version is then $\log (i / N) \approx C-\log X_{(i)}-(b /(1-\gamma)) X_{(i)}^{2-2 \gamma}$, or equivalently,

$$
\log X_{(i)} \approx C_{N}-\log i-\frac{b}{1-\gamma} X_{(i)}^{2-2 \gamma}
$$

Without the last term, the linear component in the regression of $\log X_{(i)}$ on $\log i$ has slope -1 .

(4) $\gamma=\frac{1}{2}$. In this case, $\log F(z) \cong-2 b z-(1-2 a) \log z+C$. The empirical version is $\log (i / N) \approx-2 b X_{(i)}-(1-2 a) \log X_{(i)}+C$, or equivalently,

$$
\log X_{(i)} \approx C_{N}-\frac{1}{1-2 a} \log i-\frac{2 b}{1-2 a} X_{(i)}
$$

The slope in the size distribution is $-1 /(1-2 a)$.

(5) $0<\gamma<\frac{1}{2}$. By Theorem 3.2, $\log F(z) \cong C-\log z-(b /(1-\gamma)) z^{2-2 \gamma}+(2 a /(1-$ $2 \gamma)) z^{1-2 \gamma}$. The empirical version is thus $\log (i / N) \approx C-\log X_{(i)}-(b /(1-\gamma)) X_{(i)}^{2-2 \gamma}+$ $(2 a /(1-2 \gamma)) X_{(i)}^{1-2 \gamma}$, or equivalently,

$$
\log X_{(i)} \approx C_{N}-\log i-\frac{b}{1-\gamma} X_{(i)}^{2-2 \gamma}+\frac{2 a}{1-2 \gamma} X_{(i)}^{1-2 \gamma}
$$

Since there are two nonlinear terms involved, in general the contribution from the last two terms is not small, meaning that a plot of $\log X_{(i)}$ versus the $\log$-rank $\log i$ would not display a linear pattern.

The table below summarizes the size distribution for the five different cases discussed above.

\begin{tabular}{lccccc}
\hline Cases: & $\gamma>1$ & $\gamma=1$ & $\frac{1}{2}<\gamma<1$ & $\gamma=\frac{1}{2}$ & $0<\gamma<\frac{1}{2}$ \\
Slope in the size distribution: & $-\frac{1}{2 \gamma-1}$ & $-\frac{1}{1+2 b}$ & -1 & $-\frac{1}{1-2 a}$ & Not available \\
\hline
\end{tabular}

Using the relationship (4.1), we note that the above table also gives the slope of plotting the $\log$-market-cap $\log T_{(i)}$ versus the $\log$-rank $\log i$.

REMARK 1. The case of $\frac{1}{2}<\gamma<1$ may have some interesting applications in city size distribution, as it is observed empirically that the slope of city size distribution is always very close to -1 ; see, for example, Krugman (1996) and Gabaix (1999).

\section{Size distribution for biotechnology and Internet stocks.}

5.1. Explaining the size distribution puzzle. For biotechnology and Internet stock, the empirical observation (see \$6) reveals that the slope of the size distribution is always less than -1 . Therefore, in view of the result in the table of the previous section, for biotechnology and Internet stocks $\gamma$ must be $\frac{1}{2}$ and $0<a<\frac{1}{2}$ in the diffusion (2.3). In other words,

$$
d X(t)=\left(-b \sigma^{2} X(t)+a \sigma^{2}\right) d t+\sigma \sqrt{X(t)} d W(t), \quad X(0)=x>0,0<a<\frac{1}{2},
$$

which corresponds to the Feller process also used in finance as the CIR model (Cox et al. 1985) for the spot interest rate. 
Kou and Kou: A Diffusion Model for Growth Stocks

REMARK 2. This, however, does not imply that for other growth stocks, such as railroad and utility stocks back in the 1900s, or for any new groups of growth stocks in the future, $\gamma$ must be $\frac{1}{2}$. It only says that currently for biotechnology and Internet stocks it appears that $\gamma=\frac{1}{2}$ and $0<a<\frac{1}{2}$.

Below is a brief review of some properties of this process necessary for further discussion. Let $p(t, x, y)=\mathrm{P}(X(t) \in d y \mid X(0)=x)$ be the transition density of $X(t)$. It is well known (Cox et al. 1985, Equation (18)) that

$$
\begin{aligned}
p(t, x, y)= & \frac{(2 b)^{2 a} y^{2 a-1} e^{-2 b y}}{1-e^{-\sigma^{2} b t}} \exp \left\{-\frac{2 b e^{-b \sigma^{2} t}(x+y)}{1-e^{-b \sigma^{2} t}}\right\} \\
& \cdot\left(4 b^{2} e^{-b \sigma^{2} t} x y\right)^{-(2 a-1) / 2} I_{2 a-1}\left(\frac{4 b \sqrt{x y e^{-b \sigma^{2} t}}}{1-e^{-b \sigma^{2} t}}\right),
\end{aligned}
$$

where $I_{2 a-1}(\cdot)$ is the modified Bessel function of order $2 a-1$ (for references concerning Bessel function, see Szegö 1939). From (5.2), it is seen that $p(t, x, y)$ follows a rescaled noncentral $\chi^{2}$ distribution with the moment generating function $M(\theta, t, x)=$ $\int_{0}^{\infty} e^{\theta y} p(t, x, y) d y$ satisfying

$$
M(\theta, t, x)=\left(\frac{-2 b}{-2 b+\theta-e^{\left(-b \sigma^{2}\right) t} \theta}\right)^{2 a} \exp \left\{\frac{-2 b \theta x e^{-b \sigma^{2} t}}{-2 b+\theta-\theta e^{-b \sigma^{2} t}}\right\} .
$$

The steady-state moment generating function is hence given by

$$
M(\theta)=\lim _{t \rightarrow \infty} M(\theta, t, x)=\left(\frac{-2 b}{-2 b+\theta}\right)^{2 a}, \quad \forall x>0,
$$

which is exactly the moment-generating function of the gamma distribution $\Gamma(2 a, 1 / 2 b)$. Two immediate results from (5.3) are that the transient mean and variance of $X_{t}$ are, respectively,

$$
\begin{aligned}
& m(t)=E(X(t) \mid X(0)=x)=x e^{-b \sigma^{2} t}-\frac{a}{b}\left(e^{-b \sigma^{2} t}-1\right) \stackrel{t \rightarrow \infty}{\longrightarrow} \frac{a}{b}, \\
& V(t)=\operatorname{Var}(X(t) \mid X(0)=x)=-\frac{x}{b}\left(e^{-2 b \sigma^{2} t}-e^{-b \sigma^{2} t}\right)+\frac{a}{2 b^{2}}\left(e^{-b \sigma^{2} t}-1\right)^{2} .
\end{aligned}
$$

With some properties of the process (5.1) reviewed, now we return to the size distribution study. Recall we have shown in the previous section that for large biotechnology and Internet stocks (thus satisfying Assumption (A2)),

$$
\log X_{(i)} \approx C_{N}-\frac{1}{1-2 a} \log i-\frac{2 b}{1-2 a} X_{(i)},
$$

where $C_{N}$ is a free parameter, since $N$ is unknown.

Equation (5.6) supplies a link between the ordered values of $X(t)$ and their relative ranks within the group. However, since it involves a nuisance parameter $C_{N}$, a better equation can be obtained by eliminating $C_{N}$ first, as is typical in many standard statistical procedures. This can be done by taking the difference of $\log X_{(i)}-\log X_{(1)}$ :

$$
\log \frac{X_{(i)}}{X_{(1)}} \approx-\frac{1}{1-2 a} \log i-\frac{2 b}{1-2 a}\left(X_{(i)}-X_{(1)}\right), \quad 1 \leq i \leq K .
$$

Now plugging it into (4.1) we have, for $1 \leq i \leq K$,

$$
\log \frac{T_{(i)}}{T_{(1)}} \approx-\frac{1}{1-2 a} \log i-\frac{2 b / \Theta(t)}{1-2 a}\left(T_{(i)}-T_{(1)}\right) .
$$

Equation (5.7) is the key cross-sectional empirical implication in this paper. It will lead to an "almost" line curve if the third term in (5.7) is small. This can be justified by the following lemma. 
Lemma 5.1. Suppose that $a \rightarrow 0$ and $\Theta(t)>0$. Then in the steady state, the third term in (5.7) goes to zero in $L_{1}$. In other words, under these conditions (5.7) is asymptotically a linear relationship.

Proof. It is enough to prove that

$$
\frac{2 b}{1-2 a} E\left(X_{(i)}(t)-X_{(1)}(t)\right) \longrightarrow 0, \quad 1 \leq i \leq K .
$$

Since $0 \leq X_{(i)}(t) \leq X_{(1)}(t)$, we only need to show that

$$
\frac{2 b}{1-2 a} E\left[X_{(1)}(t)\right] \longrightarrow 0 .
$$

Noting that $E\left[X_{(1)}(t)\right] \leq N \cdot E[X(t)]$, the problem is further reduced to show that

$$
\frac{2 b}{1-2 a} E[X(t)] \longrightarrow 0 .
$$

In the steady state, using (5.4) this becomes $(2 b /(1-2 a))(a / b) \rightarrow 0$, which is true because $a \rightarrow 0$.

As we shall see in $\S 6, a$ indeed tends to be small in the numerical examples, ranging from 0.06 to 0.19 . In fact, the magnitude of $a$ seems to be small enough so that the nonlinear fit in Figures 1 and 2 seems almost linear. It is worth mentioning that even if $a$ is not small, the nonlinear, cross-sectional Equation (5.7) still holds.

REMARK 3. The cross-sectional Equation (5.7) postulates a relationship between the logarithm of the normalized (by the largest value) market caps and the logarithm of the ranks of "large-cap" growth stocks (those satisfy Assumption (A2)). The word "large-cap" here is used in a loose sense and should not be confused with similar words used in stock
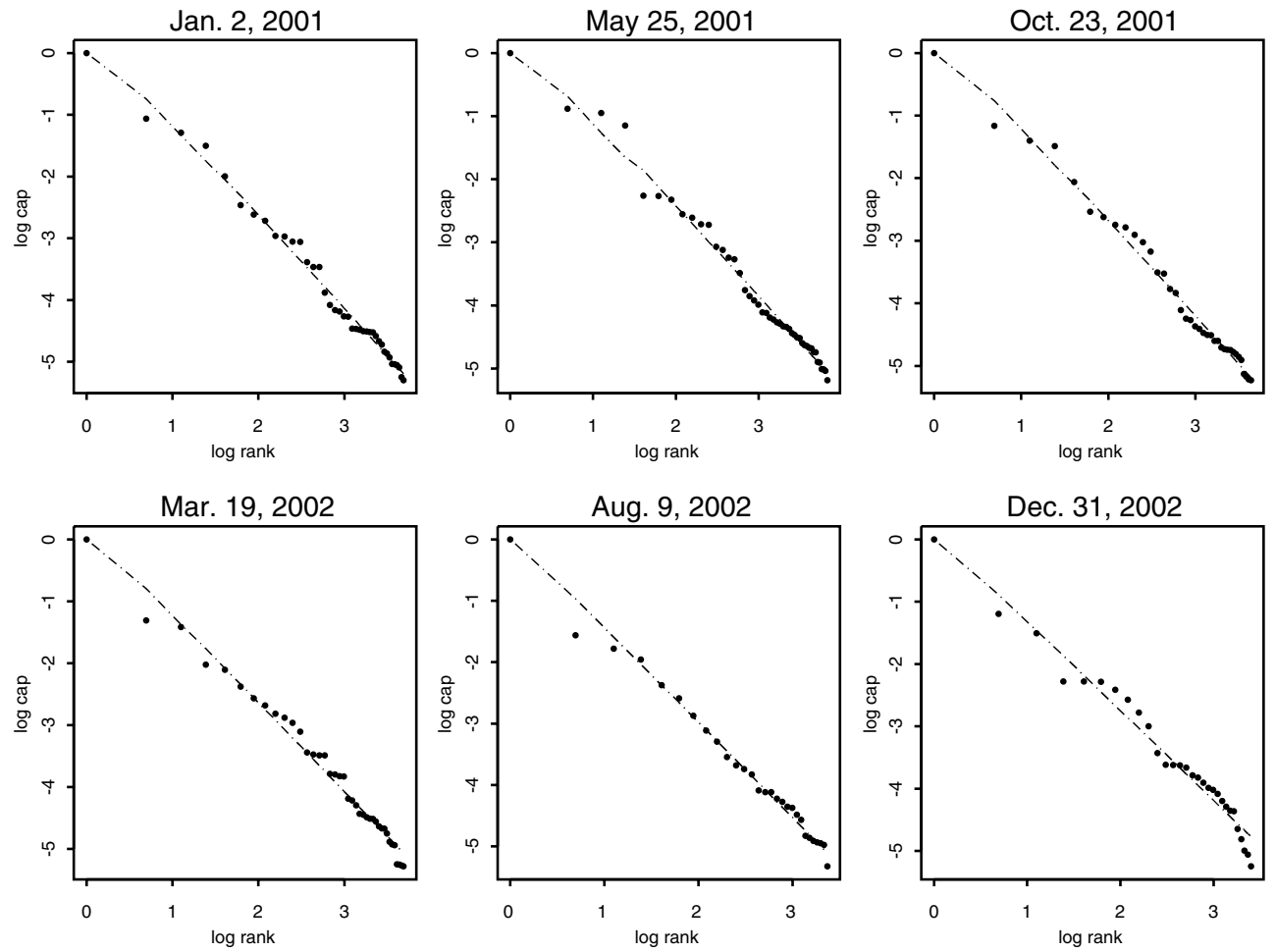

FIGURE 1. Empirical and estimated size distribution for Internet stocks. 
Kou and Kou: A Diffusion Model for Growth Stocks
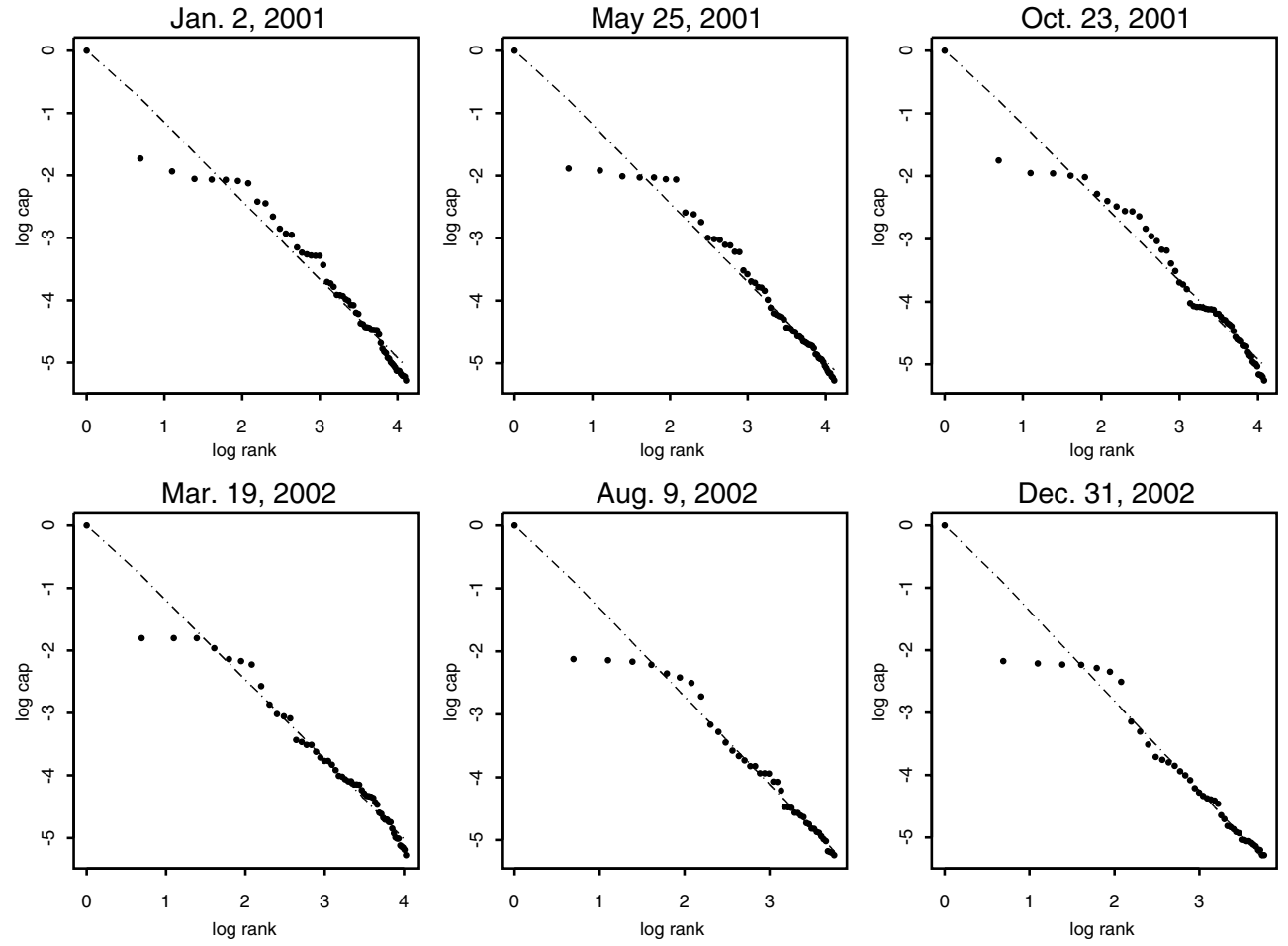

FIGURE 2. Empirical and estimated size distribution for biotechnology stocks.

exchanges; more precisely, it means that the market capitalization is large enough so that the asymptotic result for the tail distribution in Theorem 3.2, Part (4), holds.

REMARK 4. It may be worthwhile to point out the connection between the new crosssectional implication (5.7) and the empirical puzzle (only for Internet stocks) reported in the Wall Street Journal (later summarized in Maubossin and Schay 2000), which amounts to

$$
\log T_{(i)}(t) \approx a(t)+b(t) \log i
$$

where $a(t)$ and $b(t)$ do not depend on the index $i$. The new cross-sectional implication (5.7) appears to have a better fit to the data, as judged by the high $R^{2}$ statistic (see $\S 6$ ). There are two reasons for the improvement: (1) We eliminate the nuisance parameter by using the relative market caps $T_{(i)} / T_{(1)}$. (2) We do not require the third term in (5.7) to be zero or small when we fit the model, thus leading to a better fit.

5.2. The decay parameter. Using the property of steady states, we explained the size distribution puzzle. However, it is important to note that the size distribution in steady state is relevant only if the convergence from the transient states to the steady state is fast enough, i.e., if the convergence can be observed in a timely fashion. There are many ways to judge the convergence speed. One good way to evaluate the convergence speed of the whole distribution function is to look at the convergence of density functions. Therefore, we define the decay parameter, motivated by a similar definition for Markov chains in Kijima (1997), as

$$
\delta:=\sup \left\{\alpha \geq 0: p(t, x, y)-p(y)=O\left(e^{-\alpha t}\right), \forall x>0\right\},
$$

where $p(y)$ is the steady-state density function. 
Proposition 5.1. For the process in (5.1), the decay parameter $\delta=b \sigma^{2}$.

Proof. By Karlin and Taylor (1981, p. 334, Equation (13.15)), we have

$$
\begin{aligned}
p(t, x, y)= & (2 b)^{2 a} y^{2 a-1} e^{-2 b y} \sum_{n=0}^{\infty} e^{-n b \sigma^{2} t} L_{n}^{(2 a-1)} \\
& \cdot\left(\frac{b \sigma^{2} x}{\sigma^{2} / 2}\right) L_{n}^{(2 a-1)}\left(\frac{b \sigma^{2} y}{\sigma^{2} / 2}\right) \frac{\Gamma(n+1)}{\Gamma(n+2 a)},
\end{aligned}
$$

where $L_{n}^{(2 a-1)}(\cdot)$ is the Laguerre polynomial with parameter $2 a-1$ (Szegö 1939). (Note that there is a typo in the book. We thank the referee for pointing this out.) Since $L_{0}^{(2 a-1)}(\cdot) \equiv 1$, the term corresponding to the term $n=0$ is exactly the steady-state density $p(y)$. For $n \geq 1$, the only term inside the summation involving $t$ is $e^{-n b \sigma^{2} t}$. Therefore, $\delta=b \sigma^{2}$.

The decay parameter calculated in Proposition 5.1 agrees well with our intuition gained from (5.3), (5.4), and (5.5) about the convergence of the moment generating function, the mean, and the variance. Note that the decay parameter $\delta$ affects the convergence in an exponential way. Therefore, a small difference in $\delta$ can have a remarkable effect on the speed of convergence, which in turn suggests that the steady-state analysis of the size distribution in our model is only relevant when the decay parameter is large.

This helps explain why the almost linear relationship between the logarithm of the market capitalization and the logarithm of the ranks does not appear for nongrowth stocks. There are at least two reasons. First, the mean reverting diffusion model may not be valid for nongrowth stocks. Second, even if the model is valid for nongrowth stocks, in order to empirically observe such a phenomenon as implied by (5.7), the convergence from the transient states to the steady state must be fast enough, which depends on the magnitude of the decay parameter $\delta$.

It is well known that the volatility for growth stocks is much larger than that of nongrowth stocks. For example, Kerins et al. (2001) show empirically that the volatility of Internet stocks is at least five times that of traditional stocks. In the model (5.1), if $\sigma$ of growth stocks is five times larger, then $\sigma^{2}$ is 25 times larger! This leads to a much larger decay rate $\delta$ (which affects the convergence in an exponential way).

Equation (5.7), therefore, also implies that the same cross-sectional phenomenon should hold not only for large-cap Internet stocks (as reported in the Wall Street Journal article) but also for other large-cap growth stocks, such as large-cap biotechnology stocks, as long as the volatility $\sigma$ is large.

For nongrowth stocks due to the slow convergence from the transient states to the steady state, the steady-state Equation (5.7) may not emerge at all within a reasonable amount of time. Furthermore, if the convergence rate is slow, many factors can lead the process to depart away from the original steady state, e.g., change of parameters in the model, etc.

5.3. Further remarks about the cross-sectional implication. Equation (5.7) provides a way to price a growth stock relative to its peers within the group (the contribution of the peer group is to provide an estimate of $a$ and $b$, and the relative ranks) by (a) first running a regression to obtain the parameters $a$ and $b$ subject to the constraints $a>0$ and $b>0$, and (b) once the parameters are obtained, calculating the theoretical market capitalization of the stock according to (5.7) with the input being its rank. This, subsequently, provides a theoretical value of the shares price, after dividing the market capitalization by the total number of outstanding shares.

To use the model to relatively price large-cap growth stocks, it is important to keep in mind that the stocks within the peer group should have similar parameters $a$ and $b$ (for example, it may not be sensible to mix biotechnology stocks and Internet stocks together 
Kou and Kou: A Diffusion Model for Growth Stocks

TABLE 1. Estimated parameters for biotechnology stocks from Jan. 1998 to Dec. 2000.

\begin{tabular}{lccc}
\hline & $\hat{a}$ & $\hat{\mu}$ & $R^{2}(\%)$ \\
\hline Jan. 2, 1998 & 0.057 & $6.64 \times 10^{-10}$ & 98.2 \\
Aug. 7, 1998 & 0.099 & $6.01 \times 10^{-10}$ & 98.1 \\
Mar. 15, 1999 & 0.162 & $5.06 \times 10^{-10}$ & 97.8 \\
Oct. 15, 1999 & 0.151 & $5.24 \times 10^{-10}$ & 98.9 \\
May 19, 2000 & 0.115 & $5.78 \times 10^{-10}$ & 97.5 \\
Dec. 21, 2000 & 0.112 & $8.79 \times 10^{-10}$ & 96.4 \\
\hline
\end{tabular}

as their parameters may be quite different). However, in principle, the relative pricing does not require $\sigma^{2}$ to be the same; the only requirement is that $\sigma^{2}$ must be very large, as $\sigma^{2}$ only controls the speed of convergence from transient to steady state and does not enter Equation (5.7).

6. Numerical illustration. In this section we consider fitting the size distribution implied by the diffusion model. We use least squares to estimate the parameters in (5.7):

$$
(\hat{a}, \hat{\mu})=\underset{a>0, \mu>0}{\arg \min } \sum_{i=1}^{K}\left[\log \frac{T_{(i)}}{T_{(1)}}-\left\{-\frac{1}{1-2 a} \log i-\frac{2 \mu}{1-2 a}\left(T_{(i)}-T_{(1)}\right)\right\}\right]^{2},
$$

where $\mu=b / \Theta$. Tables 1 and 2 report the estimated parameters as well as the $R^{2}$ in the fitting of six trading days for 123 biotech stocks (every 150 trading days from January 1998 to December 2000) and 61 Internet stocks in six trading days (every 100 trading days from January 1999 to December 2000). For a description of the stocks used in this illustration, see Appendix B.

Note that $\hat{\mu}$ are all very small, which hints that $\Theta$ might be quite large. Moreover the $R^{2}$ being at least $93 \%$ directly supports the prediction power of the model. For the recent market from January 2001 to December 2002 (every 100 trading days), Tables 3 and 4 report the estimated parameters and the $R^{2}$; Figures 1 and 2 plot the observed and estimated size distribution. Again the $R^{2}$ is at least $95 \%$. The fitting seems to be well even under the severe market downturn during the period. (Note that the "Internet bubble" had burst then.)

TABLE 2. Estimated parameters for Internet stocks from Jan. 1999 to Dec. 2000.

\begin{tabular}{lccc}
\hline & $\hat{a}$ & $\hat{\mu}$ & $R^{2}(\%)$ \\
\hline Jan. 4, 1999 & 0.194 & $8.27 \times 10^{-10}$ & 97.3 \\
May 27, 1999 & 0.156 & $7.70 \times 10^{-10}$ & 96.6 \\
Oct. 19, 1999 & 0.142 & $5.38 \times 10^{-10}$ & 98.6 \\
Mar. 13, 2000 & 0.076 & $5.85 \times 10^{-11}$ & 93.5 \\
Aug. 3, 2000 & 0.175 & $4.87 \times 10^{-10}$ & 98.0 \\
Dec. 26, 2000 & 0.163 & $2.43 \times 10^{-10}$ & 99.2 \\
\hline
\end{tabular}

TABLE 3. Estimated parameters for Internet stocks from Jan. 2001 to Dec. 2002.

\begin{tabular}{lccc}
\hline & $\hat{a}$ & $\hat{\mu}$ & $R^{2}(\%)$ \\
\hline Jan. 2, 2001 & 0.176 & $6.71 \times 10^{-10}$ & 99.0 \\
May 25, 2001 & 0.160 & $1.19 \times 10^{-9}$ & 98.6 \\
Oct. 23, 2001 & 0.178 & $1.23 \times 10^{-9}$ & 98.7 \\
Mar. 19, 2002 & 0.155 & $7.90 \times 10^{-10}$ & 98.3 \\
Aug. 9, 2002 & 0.179 & $4.81 \times 10^{-10}$ & 98.7 \\
Dec. 31, 2002 & 0.156 & $6.10 \times 10^{-10}$ & 97.1 \\
\hline
\end{tabular}


Kou and Kou: A Diffusion Model for Growth Stocks Mathematics of Operations Research 29(2), pp. 191-212, () 2004 INFORMS

TABLE 4. Estimated parameters for biotechnology stocks from Jan. 2001 to Dec. 2002.

\begin{tabular}{lccc}
\hline & $\hat{a}$ & $\hat{\mu}$ & $R^{2}(\%)$ \\
\hline Jan. 2, 2001 & 0.105 & $8.26 \times 10^{-10}$ & 95.9 \\
May 25, 2001 & 0.106 & $5.92 \times 10^{-10}$ & 96.3 \\
Oct. 23, 2001 & 0.101 & $5.98 \times 10^{-10}$ & 96.2 \\
Mar. 19, 2002 & 0.109 & $5.86 \times 10^{-10}$ & 97.3 \\
Aug. 9, 2002 & 0.143 & $5.36 \times 10^{-10}$ & 95.6 \\
Dec. 31, 2002 & 0.192 & $4.62 \times 10^{-9}$ & 96.3 \\
\hline
\end{tabular}

7. Discussion. In this paper, we propose a diffusion model for growth stocks, whose key feature of mean reversion supplies a useful tool to model the recent boom and burst of the "Internet bubble," which is otherwise quite difficult to explain under the traditional valuation framework.

Three attractive features of the model are: (1) It does not depend on any information about forecasted earnings or sales data. Some practitioners have used forecasted sales data from various financial analysts to predict the prices of growth stocks. Yet, there is no clear mathematical relationship between the sales data and the prices of growth stocks, and the forecasted sales data in many cases is far from reliable, as is evident during the recent burst of the "Internet bubble." (2) The model leads to a cross-sectional Equation (5.7) for growth stocks, which agrees with the empirical observations very well; in addition, since fitting the model only requires regression and relative ranks, it is easy to implement. (3) The cross-sectional implication of the model remains valid irrespective to the market ups and downs, because the model compares the relative value of a stock to the stocks within its peer group.

As is common for many cross-sectional studies, Equation (5.7) should only be viewed as an understanding of growth stocks as a whole rather than as a trading tool, because we did not provide dynamics of the relative ranks for growth stocks. However, crosssectional implications may lead to some useful economic models. In fact, it can be shown that a dynamic equilibrium model can be built as a result of the model proposed in the current paper, and the model is also linked to stochastic endogenous growth theory in macroeconomics; the details of the macroeconomic justification of the current model, being too long to be included here, are given in Kou and Kou (2002).

\section{Appendix.}

A. Proofs. To prove Theorem 2.1, we shall use the following theorem on the weak convergence of birth-death processes.

Stone's Theorem (Stone 1963, see also Iglehart 1974). Suppose that $X_{n}(t)$ is a sequence of birth-death processes with $X_{n}(0)=x_{n}$, state space $E_{n}$, and infinitesimal mean $m_{n}(x)$, and variance $\sigma_{n}^{2}(x), x \in E_{n}$. Define a diffusion process $X(t)$, with the generator

$$
\frac{1}{2} \sigma^{2}(x) \frac{d^{2}}{d x^{2}}+m(x) \frac{d}{d x}, \quad 0 \leq x<\infty,
$$

and initial value $X(0)=x$. Then $X_{n}(t) \Rightarrow X(t)$ on $D[0, \infty)$ with Stone's topology, if the following three conditions are satisfied: (a) $x_{n} \rightarrow x$; (b) $E_{n}$ becomes dense in $[0, \infty)$ as $n \rightarrow \infty$; (c) for every compact subinterval I of $[0, \infty), \lim _{n \rightarrow \infty} m_{n}\left(e_{n}(y)\right)=m(y)$ and $\lim _{n \rightarrow \infty} \sigma_{n}^{2}\left(e_{n}(y)\right)=\sigma^{2}(y)$, uniformly for $y \in I$, where $e_{n}(y):=\sup \left\{y_{i} \in E_{n}: y_{i}<y\right\}$.

Proof of Theorem 2.1. Condition (a) is clearly satisfied. For condition (b), note that $E_{n}$ consists of all the points $m / n$, for all integers $m \geq 0$; thus, the limit of $E_{n}$ will be 
Kou and Kou: A Diffusion Model for Growth Stocks

the set of all rational numbers on $[0, \infty)$, which is dense in $[0, \infty)$. Finally, the infinitesimal mean and variance of $X_{n}$ are given by

$$
\begin{array}{ll}
m_{n}(y)=\frac{\left(\lambda_{n}-\mu_{n}\right) y+\left(g_{n}-h_{n}\right)}{n}, & y \in E_{n}, \\
\sigma_{n}^{2}(y)=\frac{\left(\lambda_{n}+\mu_{n}\right) y+\left(g_{n}+h_{n}\right)}{n^{2}}, & y \in E_{n} .
\end{array}
$$

Therefore, $m_{n}\left(e_{n}(y)\right) \rightarrow-b \sigma^{2} y+a \sigma^{2}$, and $\sigma_{n}^{2}\left(e_{n}(y)\right) \rightarrow \sigma^{2} y$ both uniformly for $y \in$ $I$, any compact subinterval of $[0, \infty)$. Thus Condition (c) is satisfied, and the proof is terminated.

Proof of Lemma 3.1.

(i) Let us first consider the case $\gamma \neq 1, \frac{1}{2}$. The result of $\lim _{x \rightarrow 0+} \sigma^{2}(x) s(x)$ follows easily as

$$
\begin{aligned}
\lim _{x \rightarrow 0+} \sigma^{2}(x) s(x) & =\sigma^{2} \lim _{x \rightarrow 0+}\left\{x^{2 \gamma} \exp \left\{\frac{1}{x^{2 \gamma-2}}\left(\frac{2 a}{2 \gamma-1} \frac{1}{x}-\frac{b}{\gamma-1}\right)\right\}\right\} \\
& = \begin{cases}\infty, & \text { if } \gamma>1, \\
\infty, & \text { if } \frac{1}{2}<\gamma<1, \\
0, & \text { if } 0<\gamma<\frac{1}{2} .\end{cases}
\end{aligned}
$$

Applying L'Hôpital's rule gives

$$
\begin{aligned}
\lim _{x \rightarrow 0} \frac{S(x)}{\sigma^{2}(x) s(x)} & =\lim _{x \rightarrow 0} \frac{s(x)}{\left\{\sigma^{2}(x) s(x)\right\}^{\prime}} \\
& =\frac{1}{\sigma^{2}} \lim _{x \rightarrow 0} \frac{\exp \left((b /(1-\gamma)) x^{2-2 \gamma}\right) \exp \left((2 a /(2 \gamma-1)) x^{1-2 \gamma}\right)}{\left\{x^{2 \gamma} \exp \left((b /(1-\gamma)) x^{2-2 \gamma}\right) \exp \left((2 a /(2 \gamma-1)) x^{1-2 \gamma}\right)\right\}^{\prime}} \\
& =\frac{1}{\sigma^{2}} \lim _{x \rightarrow 0} \frac{1}{2 \gamma x^{2 \gamma-1}+2 b x-2 a} \\
& = \begin{cases}-\frac{1}{2 a \sigma^{2}}<0, & \text { if } \gamma>1, \\
-\frac{1}{2 a \sigma^{2}}<0, & \text { if } \frac{1}{2}<\gamma<1, \\
0, & \text { if } 0<\gamma<\frac{1}{2} .\end{cases}
\end{aligned}
$$

Hence,

$$
\begin{gathered}
\lim _{x \rightarrow 0+} S(x)= \begin{cases}-\infty, & \text { if } \gamma>1, \\
-\infty, & \text { if } \frac{1}{2}<\gamma<1, \\
0, & \text { if } 0<\gamma<\frac{1}{2},\end{cases} \\
S(0, x]=S(x)-S(0+)= \begin{cases}\infty, & \text { if } \gamma>1, \\
\infty, & \text { if } \frac{1}{2}<\gamma<1, \\
<\infty, & \text { if } 0<\gamma<\frac{1}{2} .\end{cases}
\end{gathered}
$$

For $M(0, y]$ note that

$$
\begin{aligned}
M(0, y] & =\int_{0}^{y} \frac{1}{\sigma^{2}(x) s(x)} d x \\
& =\frac{1}{\sigma^{2}} \int_{0}^{y}\left\{x^{2 \gamma} \exp \left\{\frac{1}{x^{2 \gamma-2}}\left(\frac{2 a}{2 \gamma-1} \frac{1}{x}-\frac{b}{\gamma-1}\right)\right\}\right\}^{-1} d x
\end{aligned}
$$




$$
= \begin{cases}<\infty, & \text { if } \gamma>1, \\ <\infty, & \text { if } \frac{1}{2}<\gamma<1, \\ \sim \int_{0}^{y} x^{-2 \gamma} d x<\infty, & \text { if } 0<\gamma<\frac{1}{2} .\end{cases}
$$

For $N(0,1]$ note that

$$
N(0,1]=\int_{0}^{1}\left[\int_{\eta}^{1} s(\xi) d \xi\right] \frac{1}{\sigma^{2}(\eta) s(\eta)} d \eta=\int_{0}^{1} \frac{S(1)-S(\eta)}{\sigma^{2}(\eta) s(\eta)} d \eta<\infty,
$$

because

$$
\int_{0}^{1} \frac{1}{\sigma^{2}(\eta) s(\eta)} d \eta<\infty, \quad \int_{0}^{1} \frac{S(\eta)}{\sigma^{2}(\eta) s(\eta)} d \eta>-\infty
$$

as

$$
\lim _{x \rightarrow 0+} \frac{S(\eta)}{\sigma^{2}(\eta) s(\eta)}=\text { const. }
$$

The result of $\lim _{x \rightarrow \infty} \sigma^{2}(x) s(x) / x$ follows easily as

$$
\begin{aligned}
\lim _{x \rightarrow \infty} \frac{\sigma^{2}(x) s(x)}{x} & =\sigma^{2} \lim _{x \rightarrow \infty}\left\{x^{2 \gamma-1} \exp \left\{\frac{1}{x^{2 \gamma-2}}\left(\frac{2 a}{2 \gamma-1} \frac{1}{x}-\frac{b}{\gamma-1}\right)\right\}\right\} \\
& =\infty, \quad \text { if } \gamma \neq \frac{1}{2}, 1 .
\end{aligned}
$$

Also, L'Hôpital's rule gives

$$
\begin{aligned}
\lim _{x \rightarrow \infty} \frac{S(x)}{\sigma^{2}(x) s(x) / x} & =\lim _{x \rightarrow \infty} \frac{S^{\prime}(x)}{\left\{\sigma^{2}(x) s(x) / x\right\}^{\prime}} \\
& =\frac{1}{\sigma^{2}} \lim _{x \rightarrow \infty} \frac{1}{(2 \gamma-1) x^{2 \gamma-2}+2 b-2 a x^{-1}} \\
& = \begin{cases}0, & \text { if } \gamma>1, \\
\frac{1}{2 b \sigma^{2}}>0, & \text { if } \frac{1}{2}<\gamma<1, \\
\frac{1}{2 b \sigma^{2}}>0, & \text { if } 0<\gamma<\frac{1}{2} .\end{cases}
\end{aligned}
$$

Since

$$
S(y)=\int^{y} \exp \left\{-\frac{b}{\gamma-1} \frac{1}{x^{2 \gamma-2}}+\frac{2 a}{2 \gamma-1} \frac{1}{x^{2 \gamma-1}}\right\} d x,
$$

we have

$$
\lim _{x \rightarrow \infty} S(x)=\infty, \quad \text { if } \gamma \neq \frac{1}{2}, 1 .
$$

Hence, $S[x, \infty)=\infty$, if $\gamma \neq \frac{1}{2}, 1$. Furthermore,

$$
\begin{aligned}
\int_{y}^{\infty} \frac{1}{\sigma^{2}(x) s(x)} d x & =\frac{1}{\sigma^{2}} \int_{y}^{\infty}\left\{x^{2 \gamma} \exp \left\{\frac{1}{x^{2 \gamma-2}}\left(\frac{2 a}{2 \gamma-1} \frac{1}{x}-\frac{b}{\gamma-1}\right)\right\}\right\}^{-1} d x \\
& <\infty, \quad \text { if } \gamma \neq \frac{1}{2}, 1 .
\end{aligned}
$$

Thus, $M[x, \infty)<\infty$, if $\gamma \neq \frac{1}{2}, 1$.

For $N[1, \infty)$ note that

$$
N[1, \infty)=\int_{1}^{\infty}\left[\int_{1}^{\eta} s(\xi) d \xi\right] \frac{1}{\sigma^{2}(\eta) s(\eta)} d \eta=\int_{1}^{\infty} \frac{S(\eta)-S(1)}{\sigma^{2}(\eta) s(\eta)} d \eta
$$


Kou and Kou: A Diffusion Model for Growth Stocks

The second term inside the above integer is finite as $\int_{1}^{\infty} 1 /\left(\sigma^{2}(\eta) s(\eta)\right) d \eta<\infty$. To see what happens to the first term, note that if $\gamma>1$, then L'Hôpital's rule yields

$$
\begin{aligned}
\lim _{x \rightarrow \infty} & \frac{S(x)}{\sigma^{2}(x) s(x) /\left\{x(\log x)^{2}\right\}} \\
& =\frac{1}{\sigma^{2}} \lim _{x \rightarrow \infty} \frac{\exp \left((b /(1-\gamma)) x^{2-2 \gamma}\right) \exp \left((2 a /(2 \gamma-1)) x^{1-2 \gamma}\right)}{\left\{x^{2 \gamma-1}\left(1 /(\log x)^{2}\right) \exp \left((b /(1-\gamma)) x^{2-2 \gamma}\right) \exp \left((2 a /(2 \gamma-1)) x^{1-2 \gamma}\right)\right\}^{\prime}} \\
& =\frac{1}{\sigma^{2}} \lim _{x \rightarrow \infty} \frac{(\log x)^{2}}{x^{2 \gamma-2}\{(2 \gamma-1)-2(1 / \log x)\}+2 b-2 a(1 / x)}=0 .
\end{aligned}
$$

Therefore, when $\gamma>1$, as $x \rightarrow \infty$,

$$
\frac{S(x)}{\sigma^{2}(x) s(x)}=o\left(\frac{1}{x(\log x)^{2}}\right), \quad \int_{1}^{\infty} \frac{S(\eta)}{\sigma^{2}(\eta) s(\eta)} d \eta<\infty .
$$

In addition, since $\lim _{x \rightarrow \infty}\left(S(x) /\left(\sigma^{2}(x) s(x) / x\right)\right)=1 /\left(2 b \sigma^{2}\right)>0$, when $\frac{1}{2}<\gamma<1$ or $0<$ $\gamma<\frac{1}{2}$, we have as $x \rightarrow \infty$,

$$
\frac{S(x)}{\sigma^{2}(x) s(x)}=O(1 / x), \quad \text { either } \frac{1}{2}<\gamma<1 \text { or } 0<\gamma<\frac{1}{2} .
$$

Thus,

$$
\int_{1}^{\infty} \frac{S(\eta)}{\sigma^{2}(\eta) s(\eta)} d \eta= \begin{cases}<\infty, & \text { if } \gamma>1, \\ \infty, & \text { if } \frac{1}{2}<\gamma<1, \\ \infty, & \text { if } 0<\gamma<\frac{1}{2} .\end{cases}
$$

(ii) The case of $\gamma=1$. Observe $\lim _{x \rightarrow 0+} \sigma^{2}(x) s(x)=\sigma^{2} \lim _{x \rightarrow 0+}\left\{x^{2} x^{2 b} e^{2 a / x}\right\}=\infty$, and

$$
\begin{aligned}
\lim _{x \rightarrow 0+} \frac{S(x)}{\sigma^{2}(x) s(x)} & =\frac{1}{\sigma^{2}} \lim _{x \rightarrow 0+} \frac{S(x)}{x^{2} x^{2 b} e^{2 a / x}} \\
& =\frac{1}{\sigma^{2}} \lim _{x \rightarrow 0+} \frac{x^{2 b} e^{2 a / x}}{\left(x^{2+2 b} e^{2 a / x}\right)^{\prime}} \\
& =\frac{1}{\sigma^{2}} \lim _{x \rightarrow 0+} \frac{1}{(2+2 b) x-2 a}=-\frac{1}{2 a \sigma^{2}}<0 .
\end{aligned}
$$

Therefore, $\lim _{x \rightarrow 0+} S(x)=-\infty, S(0, x]=\infty$, if $\gamma=1$.

We also have $M(0, x]<\infty$, if $\gamma=1$, because

$$
\int_{0}^{y} \frac{1}{\sigma^{2}(x) s(x)} d x=\frac{1}{\sigma^{2}} \int_{0}^{y}\left\{x^{2+2 b} e^{2 a / x}\right\}^{-1} d x<\infty .
$$

For $N(0,1]$, note that

$$
N(0,1]=\int_{0}^{1}\left[\int_{\eta}^{1} s(\xi) d \xi\right] m(\eta) d \eta=\int_{0}^{1} \frac{S(1)-S(\eta)}{\sigma^{2}(\eta) s(\eta)} d \eta<\infty,
$$

as

$$
\int_{0}^{1} \frac{1}{\sigma^{2}(\eta) s(\eta)} d \eta<\infty, \quad \int_{0}^{1} \frac{S(\eta)}{\sigma^{2}(\eta) s(\eta)} d \eta>-\infty, \quad \lim _{x \rightarrow 0+} \frac{S(x)}{\sigma^{2}(x) s(x)}=\text { const. }
$$

Note that $\lim _{x \rightarrow \infty} \sigma^{2}(x) s(x) / x=\sigma^{2} \lim _{x \rightarrow \infty}\left\{x^{2 b+1} e^{2 a / x}\right\}=\infty$, and by L'Hôpital's rule,

$$
\begin{aligned}
\lim _{x \rightarrow \infty} \frac{S(x)}{\sigma^{2}(x) s(x) / x} & =\frac{1}{\sigma^{2}} \lim _{x \rightarrow \infty} \frac{S(x)}{x^{2 b+1} e^{2 a / x}} \\
& =\frac{1}{\sigma^{2}} \lim _{x \rightarrow \infty} \frac{S(x)}{x^{2 b+1}}=\frac{1}{\sigma^{2}} \lim _{x \rightarrow \infty} \frac{s(x)}{(2 b+1) x^{2 b}} \\
& =\frac{1}{\sigma^{2}} \lim _{x \rightarrow \infty} \frac{x^{2 b} e^{2 a / x}}{(2 b+1) x^{2 b}}=\frac{1}{(2 b+1) \sigma^{2}}>0,
\end{aligned}
$$


which also gives $S[x, \infty)=\infty$, because $S(y)=\int^{y} x^{2 b} e^{2 a / x} d x$, if $\gamma=1$. In addition, $M[x, \infty)<\infty$ if $\gamma=1$, because

$$
\int_{y}^{\infty} \frac{1}{\sigma^{2}(x) s(x)} d x=\frac{1}{\sigma^{2}} \int_{y}^{\infty}\left\{x^{2+2 b} e^{2 a / x}\right\}^{-1} d x<\infty .
$$

For $N[1, \infty)$,

$$
N[1, \infty)=\int_{1}^{\infty}\left[\int_{1}^{\eta} s(\xi) d \xi\right] m(\eta) d \eta=\int_{1}^{\infty} \frac{S(\eta)-S(1)}{\sigma^{2}(\eta) s(\eta)} d \eta .
$$

Note $\int_{1}^{\infty} 1 /\left(\sigma^{2}(\eta) s(\eta)\right) d \eta<\infty$ and $\lim _{x \rightarrow \infty} S(x) /\left(\sigma^{2}(x) s(x) / x\right)=1 /\left((2 b+1) \sigma^{2}\right)>0$. Therefore, when $\gamma=1$, as $x \rightarrow \infty$,

$$
\frac{S(x)}{\sigma^{2}(x) s(x)}=O(1 / x), \quad \int_{1}^{\infty} \frac{S(\eta)}{\sigma^{2}(\eta) s(\eta)} d \eta=\infty .
$$

Thus, $N[1, \infty)=\infty$, if $\gamma=1$.

(iii) The case of $\gamma=\frac{1}{2}$. Observe that

$$
\lim _{x \rightarrow 0+} \sigma^{2}(x) s(x)=\sigma^{2} \lim _{x \rightarrow 0+}\left\{x^{1-2 a} e^{2 b x}\right\}= \begin{cases}0, & \text { if } a \leq \frac{1}{2}, \\ \infty, & \text { if } a>\frac{1}{2},\end{cases}
$$

and

$$
\lim _{x \rightarrow 0+} S(x)=\lim _{x \rightarrow 0+} \int^{x} y^{-2 a} e^{2 b y} d y= \begin{cases}>-\infty, & \text { if } 0<a \leq \frac{1}{2}, \\ -\infty, & \text { if } a>\frac{1}{2} .\end{cases}
$$

Hence, if $\gamma=\frac{1}{2}$, then

$$
S(0, x]= \begin{cases}<\infty, & \text { if } 0<a \leq \frac{1}{2}, \\ \infty, & \text { if } a>\frac{1}{2} .\end{cases}
$$

If $0<a \leq \frac{1}{2}$, then clearly

$$
\lim _{x \rightarrow 0+} \frac{S(x)}{\sigma^{2}(x) s(x)}=-\infty
$$

as $\lim _{x \rightarrow 0+} S(x)>-\infty$ and $\lim _{x \rightarrow 0+} \sigma^{2}(x) s(x)=0$. If $a>\frac{1}{2}$, then by L'Hôpital's rule,

$$
\begin{aligned}
\lim _{x \rightarrow 0+} \frac{S(x)}{\sigma^{2}(x) s(x)} & =\frac{1}{\sigma^{2}} \lim _{x \rightarrow 0+} \frac{S(x)}{x^{1-2 a} e^{2 b x}} \\
& =\frac{1}{\sigma^{2}} \lim _{x \rightarrow 0+} \frac{x^{-2 a} e^{2 b x}}{(1-2 a) x^{-2 a} e^{2 b x}+2 b x^{1-2 a} e^{2 b x}} \\
& =-\frac{1}{(2 a-1) \sigma^{2}}<0 .
\end{aligned}
$$

Furthermore, we have $M(0, x]<\infty$, if $\gamma=\frac{1}{2}$ and $a>0$, because

$$
\int_{0}^{y} \frac{1}{\sigma^{2}(x) s(x)} d x=\frac{1}{\sigma^{2}} \int_{0}^{y} \frac{1}{x^{1-2 a}} e^{-2 b x} d x<\infty .
$$

Also, $N(0, x]<\infty$ when $\gamma=\frac{1}{2}$ and $a \leq \frac{1}{2}$, because $S(0, x]<\infty$ and $M(0, x]<\infty$ (see Karlin and Taylor 1981, Table 6.1, p. 233). To check $N(0,1]$ when $a>\frac{1}{2}$, note that

$$
N(0,1]=\int_{0}^{1}\left[\int_{\eta}^{1} s(\xi) d \xi\right] m(\eta) d \eta=\int_{0}^{1} \frac{S(1)-S(\eta)}{\sigma^{2}(\eta) s(\eta)} d \eta<\infty,
$$


because $\int_{0}^{1}\left(1 / \sigma^{2}(\eta) s(\eta)\right) d \eta<\infty$, and

$$
\int_{0}^{1} \frac{S(\eta)}{\sigma^{2}(\eta) s(\eta)} d \eta>-\infty, \quad \lim _{x \rightarrow 0+} \frac{S(x)}{\sigma^{2}(x) s(x)}=\text { const., } \quad \text { if } a>\frac{1}{2} .
$$

Observe that when $\gamma=\frac{1}{2}$,

$$
\lim _{x \rightarrow \infty} \frac{\sigma^{2}(x) s(x)}{x}=\sigma^{2} \lim _{x \rightarrow \infty}\left\{x^{-2 a} e^{2 b x}\right\}=\infty .
$$

By L'Hôpital's rule,

$$
\begin{aligned}
\lim _{x \rightarrow \infty} \frac{S(x)}{\sigma^{2}(x) s(x) / x} & =\frac{1}{\sigma^{2}} \lim _{x \rightarrow \infty} \frac{S(x)}{x^{-2 a} e^{2 b x}} \\
& =\frac{1}{\sigma^{2}} \lim _{x \rightarrow \infty} \frac{e^{2 b x} x^{-2 a}}{(-2 a) x^{-2 a-1} e^{2 b x}+2 b x^{-2 a} e^{2 b x}} \\
& =\frac{1}{2 b \sigma^{2}}>0 .
\end{aligned}
$$

In addition,

$$
\lim _{x \rightarrow \infty} S(x)=\lim _{x \rightarrow \infty} \int^{x} y^{-2 a} e^{2 b y} d y=\infty, \quad \text { if } \gamma=\frac{1}{2} .
$$

Hence, $S[x, \infty)=\infty$, if $\gamma=\frac{1}{2}$. We also have $M[x, \infty)<\infty$, if $\gamma=\frac{1}{2}$, because

$$
\int_{y}^{\infty} \frac{1}{\sigma^{2}(x) s(x)} d x=\frac{1}{\sigma^{2}} \int_{y}^{\infty}\left\{x^{1-2 a} e^{2 b x}\right\}^{-1} d x<\infty .
$$

For $N[1, \infty)$, note that

$$
N[1, \infty)=\int_{1}^{\infty}\left[\int_{1}^{\eta} s(\xi) d \xi\right] m(\eta) d \eta=\int_{1}^{\infty} \frac{S(\eta)-S(1)}{\sigma^{2}(\eta) s(\eta)} d \eta .
$$

The second term is finite as

$$
\int_{1}^{\infty} \frac{S(1)}{\sigma^{2}(\eta) s(\eta)} d \eta=S(1) \int_{1}^{\infty} \frac{1}{\sigma^{2}(\eta) s(\eta)} d \eta<\infty
$$

Furthermore,

$$
\lim _{x \rightarrow \infty} \frac{S(x)}{\sigma^{2}(x) s(x) / x}=\frac{1}{2 b \sigma^{2}}>0 .
$$

Therefore, as $x \rightarrow \infty$,

$$
\frac{S(x)}{\sigma^{2}(x) s(x)}=O\left(\frac{1}{x}\right), \quad \int_{1}^{\infty} \frac{S(\eta)}{\sigma^{2}(\eta) s(\eta)} d \eta=\infty, \quad \gamma=\frac{1}{2} .
$$

Thus, $N[1, \infty)=\infty$, if $\gamma=\frac{1}{2}$. The proof is terminated.

Proof of Theorem 3.1. The table of boundary classification follows from the results in Lemma 3.1 and Table 6.2 in Karlin and Taylor (1981, p. 234).

Because the drift function $\left(-b \sigma^{2} x+a \sigma^{2}\right)$ and the volatility $\sigma(x)=\sigma x^{\gamma}(t)$ are all continuously differentiable functions in $x$ and $\sigma^{2}(x)>0$ on $(0, \infty)$, the local Lipschitz and growth conditions are satisfied (although the global Lipschitz condition may not hold). Ait-Sahalia (1996, p. 551) points out that this is enough to guarantee a unique strong solution up to the explosion time (the time to reach either 0 or $\infty$ ); this is true because of the pathwise uniqueness (by the local Lipschitz condition) combined with the existence of a weak solution up to the explosion time. 
For the four cases of (A) $\gamma>1$, (B) $\gamma=1$, (C) $\frac{1}{2}<\gamma<1$, and (D) $\gamma=\frac{1}{2}$ and $a>\frac{1}{2}$, since both 0 and $\infty$ are inaccessible, the explosion time is infinite almost surely. Therefore, the solution is unique on $[0, \infty)$. For the remaining two cases of (E) $\gamma=\frac{1}{2}$ and $0<a \leq \frac{1}{2}$, and (F) $0<\gamma<\frac{1}{2}$, one has to specify a particular boundary behavior (Karlin and Taylor 1981, pp. 239,251 ) at the boundary 0 , as 0 is the regular boundary (and is therefore accessible), to make sure the solution is unique on $[0, \infty)$. In our approach we impose 0 as a reflecting boundary (see, e.g., Mandl 1968, p. 67, and Chapter 8 in Chung and Williams 1990). With this boundary specification, the weak solution can be extended beyond the explosion time and, hence, the unique strong solution is also extended beyond the explosion time, thanks to the pathwise uniqueness due to the local Lipschitz condition (Karatzas and Shreve 1991, Theorem 2.5, p. 287).

Next, we try to study the steady-state distributions. For the cases of (A) $\gamma>1$, (B) $\gamma=1$, (C) $\frac{1}{2}<\gamma<1$, and (D) $\gamma=\frac{1}{2}$ and $a>\frac{1}{2}$, because $M(0, x]<\infty$, and $M[x, \infty)<\infty$, there exists a unique stationary distribution independent of the initial position $x$ by Theorem 7 on p. 90 in Mandl (1968). The form of the steady-state density in these cases follows from Theorem 7 on p. 90 in Mandl (1968). For the cases of (E) $\gamma=\frac{1}{2}$ and $0<a \leq \frac{1}{2}$, and (F) $0<\gamma<\frac{1}{2}$, since $M(0, x]<\infty$, and $M[x, \infty)<\infty$, Theorem 6 on p. 85 in Mandl (1968) (with $\vartheta_{0}=0, \pi_{0}=1, \sigma_{0}=0$ there, along with the results on p. 67 and Equation (9) on p. 75 of the book) implies that if we impose 0 as a reflecting boundary, then there exists a unique stationary distribution independent of the initial position $x$. The steady-state density follows from Equation (59) on p. 85 in Mandl (1968) (with $\vartheta_{0}=0, \pi_{0}=1, \sigma_{0}=0$ there). The proof is thus terminated.

Proof of TheOREM 3.2. We consider five cases.

(1) $\gamma>1$. In this case, by Theorem 3.1,

$$
f(x)=C_{2} x^{-2 \gamma} \exp \left\{2\left(\frac{b}{2 \gamma-2} x^{2-2 \gamma}-\frac{a}{2 \gamma-1} x^{1-2 \gamma}\right)\right\} .
$$

For large $x, f(x) \cong C_{2} x^{-2 \gamma}$, and the tail probability is $F(z)=\mathrm{P}(X(\infty)>z) \cong C z^{1-2 \gamma}$.

(2) $\gamma=1$. In this case, $f(x)=C_{2} x^{-2(1+b)} e^{-2 a / x}$. Note that for large $x, f(x) \cong C_{2} x^{-2-2 b}$. It follows easily that the tail probability $F(z) \cong C z^{-1-2 b}$.

(3) $\frac{1}{2}<\gamma<1$. Therefore,

$$
f(x)=C_{2} x^{-2 \gamma} \exp \left(-\frac{b}{1-\gamma} x^{2-2 \gamma}\right) \exp \left(-\frac{2 a}{2 \gamma-1} x^{1-2 \gamma}\right) .
$$

For large $x, f(x) \cong C x^{-2 \gamma} \exp \left(-(b /(1-\gamma)) x^{2-2 \gamma}\right)$. To see the tail probability, we need the following result:

$$
\int_{z}^{\infty} x^{-k} \exp \left(-\beta x^{c}\right) d x \cong \frac{1}{c \beta} z^{1-k-c} \exp \left(-\beta z^{c}\right), \quad \beta>0, c>0 .
$$

To show this, note that a change of variable $y=\beta x^{c}$ gives

$$
\begin{aligned}
\int_{z}^{\infty} x^{-k} \exp \left(-\beta x^{c}\right) d x & =\frac{1}{c}\left(\frac{1}{\beta}\right)^{(1-k) / c} \int_{\beta z^{c}}^{\infty} y^{(1-k) / c-1} e^{-y} d y \\
& \cong \frac{1}{c}\left(\frac{1}{\beta}\right)^{(1-k) / c}\left(\beta z^{c}\right)^{(1-k) / c-1} \exp \left(-\beta z^{c}\right)=\frac{1}{c \beta} z^{1-k-c} \exp \left(-\beta z^{c}\right),
\end{aligned}
$$

where the last line uses the following asymptotic property of the incomplete gamma function

$$
\int_{z}^{\infty} e^{-x} x^{k-1} d x=e^{-z} z^{k-1}\left(1+O\left(\frac{1}{z}\right)\right) .
$$

By (A.1), the tail probability is

$$
F(z) \cong C \frac{1-\gamma}{b(2-2 \gamma)} z^{-1} \exp \left(\frac{b}{\gamma-1} z^{2-2 \gamma}\right) .
$$


Kou and Kou: A Diffusion Model for Growth Stocks

(4) $\gamma=\frac{1}{2}$. This is the well-known square-root process, whose steady-state distribution is known to be a gamma distribution: $f(x)=C_{2} e^{-2 b x} x^{2 a-1}$. It follows that the tail probability $F(z) \cong C e^{-2 b z} z^{2 a-1}$. Moreover $C_{2}=C=(2 b)^{2 a} / \Gamma(2 a)$.

(5) $0<\gamma<\frac{1}{2}$. In this case,

$$
f(x)=C_{2} x^{-2 \gamma} \exp \left(-\frac{b}{1-\gamma} x^{2-2 \gamma}\right) \exp \left(-\frac{2 a}{2 \gamma-1} x^{1-2 \gamma}\right) .
$$

For $0<\gamma<\frac{1}{2}$, the tail probability is given by

$$
F(z) \cong C z^{-1} \exp \left(-\frac{b}{1-\gamma} z^{2-2 \gamma}\right) \exp \left(-\frac{2 a}{2 \gamma-1} z^{1-2 \gamma}\right) \text {. }
$$

This can be seen by using L'Hôpital's rule again.

$$
\begin{aligned}
\lim _{z \rightarrow \infty} & \frac{F(z)}{z^{-1} \exp \left(-(b /(1-\gamma)) z^{2-2 \gamma}\right) \exp \left(-(2 a /(2 \gamma-1)) z^{1-2 \gamma}\right)} \\
& =\lim _{z \rightarrow \infty} \frac{-f(z)}{\left\{z^{-1} \exp \left(-(b /(1-\gamma)) z^{2-2 \gamma}\right) \exp \left(-(2 a /(2 \gamma-1)) z^{1-2 \gamma}\right)\right\}^{\prime}} \\
& =C_{2} \lim _{z \rightarrow \infty} \frac{-1}{-z^{2 \gamma-2}-2 b+2 a z^{-1}}=\frac{C_{2}}{2 b} .
\end{aligned}
$$

The proof is terminated.

B. Data description. Except for the stocks (e.g., non-U.S. stocks) that are not included in the Center for Research in Security Prices (CRSP) historical database and the stocks no longer exist because of merger or bankruptcy, we use all the biotechnology stocks included in the NASDAQ Biotech Index (IXBT) and the Amex Biotech Index (BTK); all the Internet stocks included in the Amex Internet Index (IIX), the Dow Jones Composite Internet Index (DJINET), the Street.com Internet Index (DOT), the Amex Internet Infrastructure HOLDRS (IIH), the Amex B2B Internet HOLDRS (BHH), and the Amex Internet HOLDRS (HHH).

The total market capitalization of the stocks are first computed by taking the product of the number of outstanding shares and the share price; then the stocks with a market capitalization not smaller than $0.5 \%$ of that of the largest stock are plotted. This is because the size distribution relationship requires large market capitalization, and here "large-cap" are ad hoc adopted as stocks having market capitalization at least as large as $0.5 \%$ of that of the largest stock. One advantage of categorizing "largeness" relatively is that it automatically takes into account that different groups of stocks could have different sizes (for example, even within growth stocks, Internet stocks tend to be larger than biotechnology stocks).

To save space, instead of the full names, we only list the stocks by their ticket symbols.

\begin{tabular}{llllllllll}
\hline \multicolumn{10}{c}{ Internet stocks } \\
\hline adbe & agil & akam & amtd & amzn & artg & beas & brcm & bvsn & ckfr \\
cmgi & cmrc & coms & cs & csco & dclk & ebay & et & epny & fmkt \\
goto & hlth & homs & icge & imgx & inap & inkt & insp & intu & issx \\
itwo & iwov & jnpr & kana & lvlt & macr & navi & neta & novl & pegs \\
pcln & prsf & q & qcom & qrsi & retk & rnwk & rsas & sgi & spln \\
sqst & sunw & tibx & tmcs & tmpw & vert & vign & vitr & vntr & vrsn \\
yhoo & & & & & & & & & \\
\hline
\end{tabular}


Kou and Kou: A Diffusion Model for Growth Stocks Mathematics of Operations Research 29(2), pp. 191-212, () 2004 INFORMS

\begin{tabular}{lllllllllll}
\hline \multicolumn{10}{c}{ Biotechnology stocks } \\
\hline adrx & aimm & alks & alxn & amgn & amln & anik & apht & aria & arql & astm \\
atrx & avgn & avii & avxt & bcii & bcrx & bgen & blsi & blud & bste & btgc \\
btrn & carn & cbst & cege & ceph & cers & cgpi & chir & clgy & cnct & crxa \\
ctic & cvas & cvtx & cypb & cyph & cyto & cytr & dcrn & dige & dsco & dusa \\
emis & enmd & enzn & epix & gene & genz & gern & gild & glfd & gnlb & gnta \\
gztc & heph & hgsi & hysq & iart & iccc & icos & idph & ilxo & imcl & imgn \\
immu & imnr & incy & inhl & inkp & ipic & isip & kosp & lgnd & ljpc & lynx \\
mcde & medi & medx & mlnm & mogn & mygn & nabi & nbix & neot & nerx & nfld \\
novn & npro & npsp & nrgn & onxx & orph & osip & oxgn & pars & pcyc & pdli \\
pgnx & regn & rgen & rzym & sang & scio & scln & scri & sepr & sero & snap \\
snus & supg & teva & tgen & tktx & trms & vicl & vion & virs & vphm & vrtx \\
vvus & zona & & & & & & & & & \\
\hline
\end{tabular}

Acknowledgments. We are very grateful to an anonymous referee and Vadim Linetsky for many helpful comments, which substantially improve the presentation of the paper. A six-page summary of a preliminary version of the paper appeared in the Proceedings of the 2002 Winter Simulation Conference. This research is supported in part by NSF Grants DMS-0204674, DMS-0074637, and DMI-0216979.

\section{References}

Aït-Sahalia, Y. 1996. Nonparametric pricing of interest rate derivative securities. Econometrica 64 527-560.

Chung, K. L., R. J. Williams. 1990. An Introduction to Stochastic Integration, 2nd ed. Birkhauser, Boston, MA.

Cox, J., J. Ingersoll, S. Ross. 1985. A theory of the term structure of interest rates. Econometrica 53 385-407.

Feller, W. 1951. Two singular diffusion problems. Ann. Math. 54 173-182.

Gabaix, X. 1999. Zipf's law for cities: An explanation. Quart. J. Econom. 154 739-767.

Iglehart, D. 1974. Weak convergence in applied probability. Stochastic Processes Their Appl. 2 211-241.

Ijiri, Y., H. A. Simon. 1977. Skew Distributions and the Sizes of Business Firms. North-Holland Publishing Company, Amsterdam, The Netherlands.

Karatzas, I., S. Shreve. 1991. Brownian Motion and Stochastic Calculus, 2nd ed. Springer, New York.

Karlin, S., H. Taylor. 1981. A Second Course in Stochastic Processes. Academic Press, New York.

Kerins, F., J. K. Smith, R. Smith. 2001. New venture opportunity cost of capital and financial contracting. Working paper, Washington State University, Pullman, WA.

Kijima, M. 1997. Markov Processes for Stochastic Modeling. Chapman and Hall, London, U.K.

Kou, S. C., S. G. Kou. 2002. A tale of two growths: Modeling stochastic endogenous growth and growth stocks. Preprint. Harvard University, Boston, MA, and Columbia University, New York.

Kou, S. C., S. G. Kou. 2003. Modeling growth stocks via birth-death processes. Adv. Appl. Probab. 35 641-664.

Krugman, P. 1996. The Self-Organizing Economy. Blackwell, Cambridge, MA.

Mauboussin, M. J., A. Schay. 2000. Still powerful: The Internet's hidden order. Equity research report. Credit Suisse First Boston Corporation, July 7, 2000.

Mandl, P. 1968. Analytical Treatment of One-Dimensional Markov Processes. Springer-Verlag, Berlin, Germany and Academia, Prague, Czechoslovakia.

Pareto, V. 1896. Cours d'Economie Politique. Geneva, Switzerland.

Szegö, G. 1939. Orthogonal Polynomials. AMS Colloquium Publications, vol. 23. American Mathematical Society, Providence, RI.

Simon, H. A. 1955. On a class of skew distributions. Biometrika 42 425-440.

Stone, C. 1963. Limit theorems for random walks, birth and death processes, and diffusion processes. Illinois J. Math. 7 638-660.

Wong, E. 1964. The construction of a class of stationary Markoff processes. Sixteenth Sympos. in Appl. Math.Stochastic Processes in Math. Physics and Engrg. R. Bellman, ed. American Mathematical Society, Providence, RI, 264-276.

Yule, G. U. 1924. A mathematical theory of evolution, based on the conclusions of Dr. J. R. Willis, F.R.S. Philos. Trans. B 213 21-83.

Zipf, G. 1949. Human Behavior and the Principle of Least Effort. Addison-Wesley Press, Cambridge, MA. 